\title{
Concomitant Thymic Carcinoma With Cervical and Supraclavicular Node Metastases and Thyroid Carcinoma: Report of a Case and Literature Review
}

\author{
Stergios Doumas ${ }^{\mathrm{a}}$, Ioannis A. Voutsadakis ${ }^{\mathrm{b}, \mathrm{d}}$, Nikolaos Fourkas ${ }^{\mathrm{c}}$, \\ Maria Papagiannic, Christos N. Papandreou ${ }^{\mathrm{c}}$
}

\begin{abstract}
Thyroid and thymus neoplasms are comparatively rare. In this case report, the concomitant diagnosis of these two rare neoplasms in the same patient is presented. An increased incidence of second malignancies in patients with thymic neoplasms has been found in several case series but is still debated by others. It appears that this increased risk for second malignancies in patients with thymic neoplasms extends to a wide range of localizations and concerns neoplasms diagnosed before, concomitantly with and after thymic neoplasms. A pathogenic hypothesis of decreased immunologic surveillance is proposed and implications the association has for patient care are discussed.
\end{abstract}

Keywords: Thymoma; Thymic carcinoma; Thyroid carcinoma; Incidence; Risk; Second malignancies

\section{Introduction}

Thymic neoplasms are rare tumors accounting for $0.2 \%$ to $1.5 \%$ of all malignancies, and thymic carcinomas (TCs) represent approximately $0.06 \%$ of all malignancies $[1,2]$. According to SEER data, their incidence is 0.13 per 100,000 person-years, with a decreasing trend during the last 13 years

Manuscript accepted for publication February 7, 2012

${ }^{\mathrm{a}}$ Maxilofacial Unit, Queen Victoria Hospital, East Grinstead, UK

${ }^{\mathrm{b}}$ Department of Medical Oncology, University Hospital of Lausanne, Lausanne, Switzerland

${ }^{c}$ Department of Medical Oncology, University Hospital of Larissa, Larissa, Greece

${ }^{\mathrm{d} C}$ Corresponding author: Ioannis A. Voutsadakis, Centre Pluridisciplinaire d'Oncologie, BH06, Centre Hospitalier Universitaire Vaudois, Bugnon 46, Lausanne 1011, Switzerland.

Email: ivoutsadakis@yahoo.com

doi:10.4021/jmc568w
[3]. Nevertheless, they represent the most common masses of the anterior mediastinum. Almost $30 \%$ of anterior mediastinal tumors and $15 \%$ of their pediatric counterparts are thymomas [4]. Unusual locations such as posterior mediastinum or lower neck (ectopic thyroid thymoma) have been described [5].

Thymomas are slightly more common in males and their prevalence increases with advanced age, reaching a peak during the seventh decade of life [3]. Others have found a peak incidence during the sixth and seventh decade of life [6]. Their clinical presentation and prognosis depend on histology and stage. Indeed, thymomas are a diverse group of epithelial neoplasms with a natural history spectrum that extends from indolent to lethal thymic carcinomas. A third of them are discovered serendipitously due to the frequent utilization of chest radiographs or CT for screening purposes or for imaging of other conditions. The remainder present clinically with symptoms due to invasion or compression of intrathoracic structures, distant metastases, or paraneoplastic syndromes [3]. One third of thymoma patients will present with an invasive or advanced unresectable disease [7].

The coexistence of thymomas with numerous paraneoplastic syndromes and second primary cancers is well documented. Indeed, several studies have corroborated that thymomas are associated with one or more non-thymic neoplasms with a reported incidence rate of up to $33 \%$ [8].

In this context, we present a case of a voluminous thymic carcinoma extending to the thyroid region with neck node metastases. Interestingly, a thyroid papillary carcinoma was also concomitantly diagnosed.

\section{Case Report}

A 42-year old woman presented with a midline cervical mass and worsening dyspnea. She noticed that the lesion was rapidly growing. Past medical history was unremarkable. Physical examination revealed an ill-defined mass in the thyroid gland area extending downwards to the thoracic inlet. Enlarged cervical and supra-clavicular nodes were also noticed. A contrast-enhanced CT-scan confirmed the aforementioned clinical findings, and additionally revealed that this heteroge- 


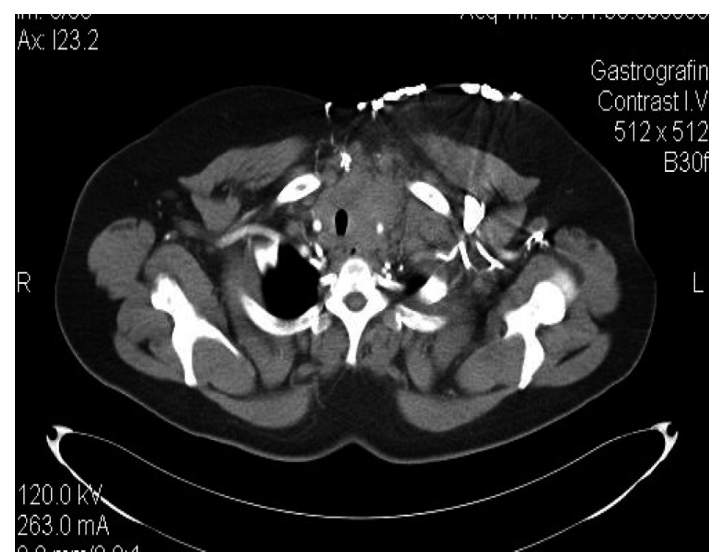

Figure 1. III defined heterogeneous, soft tissue mass occupying the anterior mediastinum. The lesion abuts mediastinal structures and displaces the trachea.

neous, space-occupying mass, measuring 5.7 to $6.6 \mathrm{~cm}$ was encroaching the anterior mediastinum structures and displacing the trachea (Fig. 1). Moreover enlarged paratracheal and prevascular lymph nodes were found. A total thyroidectomy with neck dissection was performed. Pathology of the thyroid revealed papillary thyroid carcinoma, but cervical node pathology was consistent with a thymic carcinoma (WHO type C) with neuroendocrine features. Cancer cells were positive for EMA, AE1/AE3, p53, TTF-1, NSE and Ki-67, cKit was also over-expressed in some cells, while staining for CD56, chromogranin and synaptophysin were negative. Hence, the coexistence of two neoplasms was established; an indolent thyroid cancer along with an aggressive stage $\mathrm{IVb}$ thymic carcinoma. Serum tumor marker NSE was $4.7 \mathrm{ng} / \mathrm{ml}$ (normal range 0 - $17 \mathrm{ng} / \mathrm{ml}$ ) and CYFRA 21-1 was increased at $60.9 \mathrm{ng} / \mathrm{ml}$ (normal range $0-3.3 \mathrm{ng} / \mathrm{ml}$ ). Further diagnostic workup including bronchoscopy and scintigraphy were negative. Given the neuroendocrine features of her thymic carcinoma, patient was initially treated with three cycles of cisplatin and etoposide without a response.

Three cycles of CAP-P regimen (cisplatin $50 \mathrm{mg} / \mathrm{m}^{2}$, adriamycin $50 \mathrm{mg} / \mathrm{m}^{2}$, cyclophosphamide $500 \mathrm{mg} / \mathrm{m}^{2}$, prednisone $100 \mathrm{mg} /$ day every three weeks) were administered. Treatment was well tolerated and produced an objective partial response after the 2nd cycle. At that time, adjuvant radiation therapy was recommended, but the patient declined further treatment. Three months later, she complained of headache. A brain CT showed several ring enhancing lesions that displaced the midline structures. Four of them involved the cerebral cortex bilaterally (right frontal, parietal, occipital and left parietal). A fifth lesion was located in the left cerebellum displacing the tentorium cerebelli. No herniation was noted. The patient received palliative cranial irradiation.

At 5 months post diagnosis, hepatic and bone metastases as well as local progression were evident. Patient died of disease progression 2 months later.

\section{Discussion}

Thymomas represent a rare but also heterogeneous gamut of epithelial neoplasms with a natural history spectrum that extends from indolent thymomas to aggressive thymic carcinomas. Their clinical presentation depends on histology, stage and the existence of paraneoplastic syndromes. Increased tumor size and presence of calcifications is usually associated with invasive tumors of more advanced stage [5].

An association between thymomas and other extrathymic malignancies has been noted in several case reports and case-control studies as early as 50 years ago [9-11].

In a review of 598 patients with a thymoma diagnosis the additional, other than thymoma, cancer incidence rate was $17 \%[12,13]$. The Relative Risk of extra-thymic diseases was six times higher in patients with thymomas compared with patients with parathyroid adenomas who were used as controls.

In another series of 46 thymomas, four additional neoplasms (9\%) were found [14]. Papillary thyroid carcinomas accompanied with myasthenia gravis were found in two male patients. Notably, the thyroid malignancies predated the thymomas diagnosis by 1.5 and 9 years, respectively.

An evaluation of 136 thymomas diagnosed between 1957 and 1997 yielded 48 additional neoplasms in 37 patients (27\%) [15]. Eleven tumors were colorectal carcinomas followed by six cases of pulmonary tumors. Interestingly, five cases of thyroid tumors were registered. The fact that these tumors were often diagnosed prior to or concomitant with thymomas suggested that a predisposition to cancer may underlay the association rather than thymoma treatment [16].

In a case-control study the incidence of second tumors in 192 consecutive thymomas was compared with that of 1426 patients with nasopharyngeal carcinoma (NPC) [17]. Fifteen patients of the former group developed second primaries, mostly stomach adenocarcinomas followed by liver, lung and cervical cancers. One patient had a third neoplasm, but non-solid cancers were absent. None of them received chemotherapy, and in three of them the involved site was outside the radiation field. In the NPC group, only $27(2 \%)$ second primaries were found. In 24 of them irradiation was delivered and four of second tumors occurred within this field. A statistically significant association between thymic and extrathymic neoplasms was evident (Overall Risk (OR), 4.28; 95\% CI, $1.39-13.13, \mathrm{P}<0.05$ ). Patient age was the only involved factor after multivariate analysis (adjusted OR, 1.06, 95\% CI, $1.01-1.10, \mathrm{P}<0.05)$.

Recently, Gadalla et al. retrieved files for 668 thymomas from the Swedish Cancer Registry and compared them to 2,719 population-based matched controls in terms of risk for second cancers [18]. Thymoma patients had two-fold excess risk for second cancers compared with the general population, most notably: non-melanoma skin cancer [Standardized 
Incidence Ratio (SIR) $=10.6,95 \%$ confidence intervals $(\mathrm{CI})$ $=6.0$ - 17.3], non-Hodgkin lymphoma $(\mathrm{SIR}=6.8,95 \% \mathrm{CI}=$ $3.00-13.0)$, and cervical ( $\mathrm{SIR}=6.9,95 \% \mathrm{CI}=1.4-20.1)$, endocrine $(\mathrm{SIR}=4.7,95 \% \mathrm{CI}=1.3-12.0$ ), and prostate $(\mathrm{SIR}=3.0,95 \% \mathrm{CI}=1.7-4.8)$ cancers.

In a study using SEER (Surveillance Epidemiology and End-Results) data from the period between 1973 and 1998, and in which 733 patients with thymoma were identified and compared with the general population, a SIR of 1.5 (CI 1.2 1.9) was reported [19]. A similar observed to expected ratio of 1.54 (95\% CI 1.2 - 1.9) was reported in another report based on SEER data of a more extended period [20]. These reports find excess risk for only a sub-set of cancer types such as non-Hodgkin lymphoma, sarcoma and digestive tract cancers. Nevertheless the number of individual cancer localization cases is small and no definitive conclusions can be made concerning this point. Moreover SEER data tend to under-estimate incidences as noted by the authors and this may be a cause of the difference of excess risk between these reports and reports from individual centers. SEER data could also under-estimate risk of second malignancies by including mainly cases of more malignant thymomas which, due to greater mortality, display less years at risk [21].

Few other case reports describe the asociation of thyroid carcinomas with thymomas $[16,22]$. An additional feature in our case was thymoma positivity for transcription factor TTF1 (Thyroid Transcription Factor 1, otherwise known as NKX2-1, NK2 Homeobox 1). This is an antigen usually positive in thyroid and lung cancers but very rarely in thymoma [23]. Nevertheless, TTF1 positivity is more commonly seen in thymic carcinomas with neuroendocrine features such as our patient [24].

A plausible pathogenic explanation of the association of thymomas with other cancers taking into account both the facts that associated cancers occur before, concomitant with or after the diagnosis of thymomas and are independent of thymoma treatments, relates to immunologic cancer surveillance. Thymus is an important organ for $\mathrm{T}$ cell precursors maturation into both cytotoxic and helper $\mathrm{T}$ cells as well as into $\mathrm{T}$ regulatory cells (Tregs). In this organ double negative (CD4-, CD8-) T cell precursors from the bone marrow interact with cortical and medullary thymocytes and are presented peripheral foreign and self antigens [25]. Normally, during their maturation process they become double positive and finally single positive CD4 or CD8 T cells. Cells with auto-reactive T cell receptors (TCRs) are deleted and only cells with TCRs recognizing foreign antigens are allowed to become functional cytotoxic cells. T regulatory cells with the CD25+, Foxp3+ phenotype are also produced and function to restrict immune responses and to keep in control in periphery cytotoxic cells with weakly auto-reactive receptors that have escaped deletion in thymus [26]. In thymomas, neoplastic thymocytes may display impaired physiologic function and may be less supportive of the production of cytotoxic $\mathrm{T}$ cells with cancer antigen specificity or alternatively may favor production of Tregs antagonizing these cytotoxic $\mathrm{T}$ cells.

The association of thymic neoplasms with other cancers highlighted by our case report has two important implications for the optimal care of patients with these neoplasms. First a close lifelong follow-up and cancer screening is needed for these patients especially after successful treatment of thymic neoplasms. Second one has always to keep in mind that second cancers can present concomitantly with thymic neoplasms and thus clinical suspicion and a low threshold for obtaining additional biopsies is advisable.

\section{References}

1. de Jong WK, Blaauwgeers JL, Schaapveld M, Timens W, Klinkenberg TJ, Groen HJ. Thymic epithelial tumours: a population-based study of the incidence, diagnostic procedures and therapy. Eur J Cancer. 2008;44(1):123-130.

2. Chahinian AP, Marchevsky AM. Thymomas and Thymic Tumors. In: Kufe DW, Frei III E, Holland JF, et al, editors. In: Holland-Frei Cancer Medicine, 8th edition. PMPH-USA, Shelton, CT, 2010. p. 1053-1061.

3. Engels EA. Epidemiology of thymoma and associated malignancies. J Thorac Oncol. 2010;5(10 Suppl 4):S260-265.

4. Cameron RB, Loehrer PJ, Thomas CR Jr. Neoplasms of the Mediastinum. In: DeVita VT, Lawrence TS, Rosenberg SA, editors. In: DeVita, Hellman, Rosenberg's Cancer: Principles and Practice of Oncology, 8th edition. Lippincott Williams \& Wilkins Philadelphia, PA, 2008. p. 973-988.

5. Marom EM. Imaging thymoma. J Thorac Oncol. 2010;5(10 Suppl 4):S296-303.

6. Strobel P, Hohenberger P, Marx A. Thymoma and thymic carcinoma: molecular pathology and targeted therapy. J Thorac Oncol. 2010;5(10 Suppl 4):S286-290.

7. Schmitt J, Loehrer PJ, Sr. The role of chemotherapy in advanced thymoma. J Thorac Oncol. 2010;5(10 Suppl 4):S357-360.

8. Welsh JS, Wilkins KB, Green R, Bulkley G, Askin F, Diener-West M, Howard SP. Association between thymoma and second neoplasms. JAMA. 2000;283(9):1142-1143.

9. Scannell JG. Case Records of the Massachusetts General Hospital: Case 14-1962: Pulmonary disease after removal of thymoma. N Engl J Med 1962; 266:458-465.

10. Gerard AG, Tyler HY. Large Thymoma Associated with Bronchogenic Carcinoma. Report of a Case. Dis Chest. 1964;46:238-240.

11. Souadjian JV, Enriquez P, Silverstein MN, Pepin JM. The spectrum of diseases associated with thymoma. Coincidence or syndrome? Arch Intern Med. 1974;134(2):374379.

12. Souadjian JV, Silverstein MN, Titus JL. Thymoma and 
cancer. Cancer. 1968;22(6):1221-1225.

13. Lewis JE, Wick MR, Scheithauer BW, Bernatz PE, Taylor WF. Thymoma. A clinicopathologic review. Cancer. 1987;60(11):2727-2743.

14. LeGolvan DP, Abell MR. Thymomas. Cancer. 1977;39(5):2142-2157.

15. Wilkins KB, Sheikh E, Green R, Patel M, George S, Takano M, Diener-West M, et al. Clinical and pathologic predictors of survival in patients with thymoma. Ann Surg. 1999;230(4):562-572; discussion 572-564.

16. Welsh JS, Thurman SA, Howard SP. Thymoma and multiple malignancies: a case of five synchronous neoplasms and literature review. Clin Med Res. 2003;1(3):227-232.

17. Pan CC, Chen PC, Wang LS, Chi KH, Chiang H. Thymoma is associated with an increased risk of second malignancy. Cancer. 2001;92(9):2406-2411.

18. Gadalla SM, Rajan A, Pfeiffer R, Kristinsson SY, Bjorkholm M, Landgren O, Giaccone G. A population-based assessment of mortality and morbidity patterns among patients with thymoma. Int J Cancer. 2011;128(11):26882694.

19. Engels EA, Pfeiffer RM. Malignant thymoma in the United States: demographic patterns in incidence and associations with subsequent malignancies. Int $\mathrm{J}$ Cancer. 2003;105(4):546-551.

20. Travis LB, Boice JD, Jr., Travis WD. Second primary cancers after thymoma. Int J Cancer. 2003;107(5):868870.

21. Welsh JS. Thymoma and subsequent cancers. Int J Cancer. 2004;108(2):327.

22. Senga O, Hikita H, Kinoshita T, Hara K, Miyakawa M. Myasthenia gravis with thymoma associated with occult thyroid carcinoma. Surg Today. 1992;22(1):66-68.

23. Yan B, Seng SC, Petersson F. Thymoma with nuclear expression of thyroid transcription factor-1: a potential diagnostic pitfall on core biopsy. Appl Immunohistochem Mol Morphol. 2011;19(1):76-81.

24. Lauriola L, Erlandson RA, Rosai J. Neuroendocrine differentiation is a common feature of thymic carcinoma. Am J Surg Pathol. 1998;22(9):1059-1066.

25. Heng TS, Chidgey AP, Boyd RL. Getting back at nature: understanding thymic development and overcoming its atrophy. Curr Opin Pharmacol. 2010;10(4):425-433.

26. Josefowicz SZ, Rudensky A. Control of regulatory T cell lineage commitment and maintenance. Immunity. 2009;30(5):616-625. 